\title{
Novel Wire-Guided Scalpel to Facilitate Central Venous Catheter Insertion without a Skin Bridge
}

\author{
Jacob Jeffrey Rauchwerger ${ }^{1}$, Michael Serle ${ }^{2}$, and Jeffrey C. Astbury ${ }^{3}$ \\ 'Department of Anesthesiology, Mount Sinai South Nassau Hospital, Oceanside, NY, ${ }^{2}$ Department of Angiography, FirstHealth Moore \\ Regional Hospital, Pinehurst, NC, ${ }^{3}$ Department of Anesthesiology, Butler Health System, Butler, PA, USA
}

Central venous catheter (CVC) placement is a commonly performed procedure. More than 5 million CVC placements are performed annually in the United States, with nearly $45 \%$ of critical care patients and $8 \%$ of all inpatients requiring a CVC during their hospitalization. A novel wire-guided scalpel (GuideBlade; Ambitus Medical Supplies LLC, Oceanside, NY, USA) has recently been introduced into clinical practice. In this communication, we will describe how to use the device and discuss possible advantages associated with its routine use during CVC placement.

Key Words: Skin, Surgical incision, Vascular access devices, Wire-guided, Seldinger technique
Received May 3, 2021

Revised June 27, 2021

Accepted July 13, 2021

Published on August 6, 2021

Corresponding author: Jacob Jeffrey Rauchwerger

Department of Anesthesiology, Mount Sinai South Nassau Hospital, One Healthy Way, Oceanside, NY 11572, USA

Tel: 1-7186408343

E-mail: Jacobjeffrey74@hotmail.com https://orcid.org/000-0002-0768-0817

Copyright (c) 2021 The Korean Society for Vascular Surgery

This is an Open Access article distributed under the terms of the Creative Commons Attribution Non-Commercial License (http://creativecommons.org/licenses/by-nc/4.0) which permits unrestricted non-commercial use, distribution, and reproduction in any medium, provided the original work is properly cited.

Cite this article; Vasc Specialist Int 2021. https://doi.org/10.5758/vsi.210033

\section{INTRODUCTION}

The wire-guided scalpel (GuideBlade; Ambitus Medical Supplies LLC, Oceanside, NY, USA) is a disposable safety scalpel with an integrated beveled channel that runs along the non-cutting edge of the blade and through the scalpel handle. This beveled channel is sized to accommodate guidewires of up to 0.035 inch in diameter (Fig. 1A). The blade has been modified to allow the tip to sit flush against the loaded guidewire. In addition, the scalpel has a specially designed transparent safety sheath that allows clinicians to thread a guidewire through the beveled channel while the blade is safely covered (Fig. 1B).

\section{TECHNIQUE}

Central venous catheter (CVC) placement is typically performed using the Seldinger or modified Seldinger technique [1-3]. Regardless of the specific approach utilized, the target central vein is initially cannulated with a relatively small-
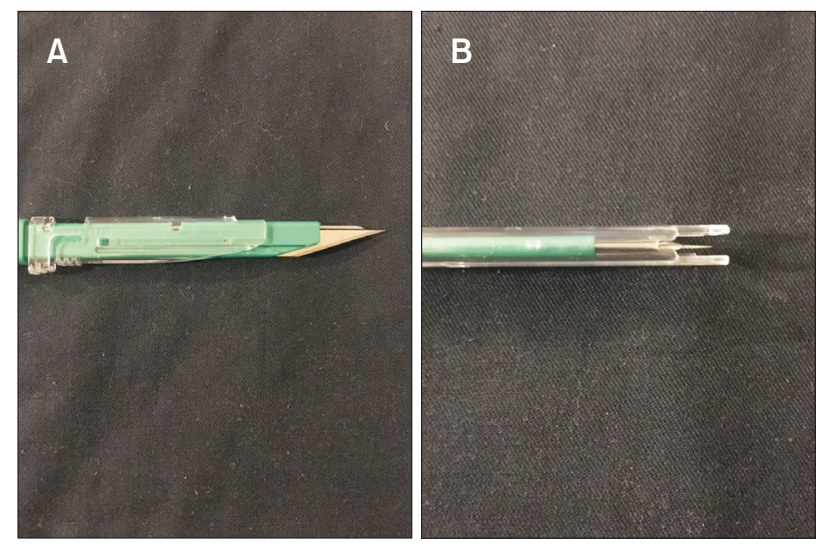

Fig. 1. (A) A wire-guided scalpel with retracted safety sheath demonstrated straight edged scalpel blade and beveled channel sized to accommodate vascular guidewires up to 0.035 inches in diameter. (B) An overhead photograph demonstrates wire-guided scalpel channel entry window with safety sheath in closed position. This feature allows clinicians to directly visualize the channel opening and easily thread the guidewire into the channel with the scalpel blade safely covered. 
bore needle. Once the vein is entered, a vascular guidewire is inserted into the target vein via the needle. The needle is removed, and the vascular wire is left in situ, with the distal end exiting the patient's skin. The wire is then used as a guide to direct the desired catheter into the target vessel. As there is typically a significant size difference between the small-bore needle used to insert the wire and the desired catheter, the wire entry site is typically expanded prior to inserting the catheter. A commonly employed approach is to make a small skin incision at the wire entry site (Fig. 2A) followed by inserting a hard plastic dilator over the wire, through the incision, and into the target vein. The dilator is then removed, and the catheter is inserted over the wire and into the vein [4].

Effective skin incisions are contiguous with the in situ guidewire, as residual skin tags or bridges between the insertion site and the incision (Fig. 2B) may make it difficult to insert dilators without using excessive force. The wire-guided scalpel utilizes an in-situ guidewire to direct

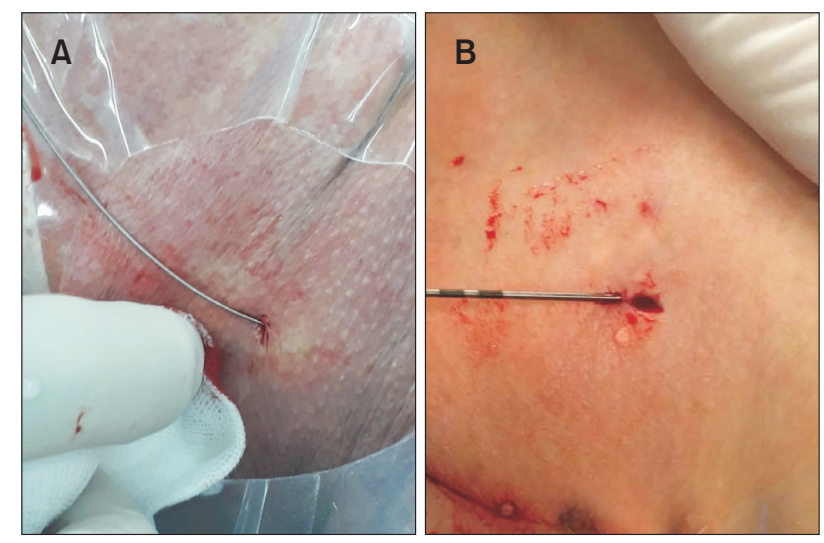

Fig. 2. (A) A properly executed skin incision. The incision is contiguous with the wire entry site with no residual skin bridges between the wire entry site and the incision. (B) A misplaced skin incision with a visible skin bridge between the wire entry site and incision. Adapted from the article of Frogel and Rauchwerger (Vasc Specialist Int 2020;36:122123) [4]. skin incision placement. With the transparent safety sheath locked in the closed position, the wire is threaded through the beveled aperture of the scalpel channel located under the blade tip and withdrawn until the tip of the wire exits the distal end of the scalpel at the end of the plastic handle (Fig. 3A). The safety sheath is then moved into the open position and the scalpel is advanced down the wire and through the skin to the desired depth (Fig. 3B) and then removed from the wire. This results in an in situ wire with a skin incision contiguous with the wire insertion site.

\section{DISCUSSION}

Skin incision is a critical step in safe CVC placement, which is sometimes overlooked. Even under ideal conditions, placing an incision that is contiguous with the wire entry site is challenging because of the small diameter of

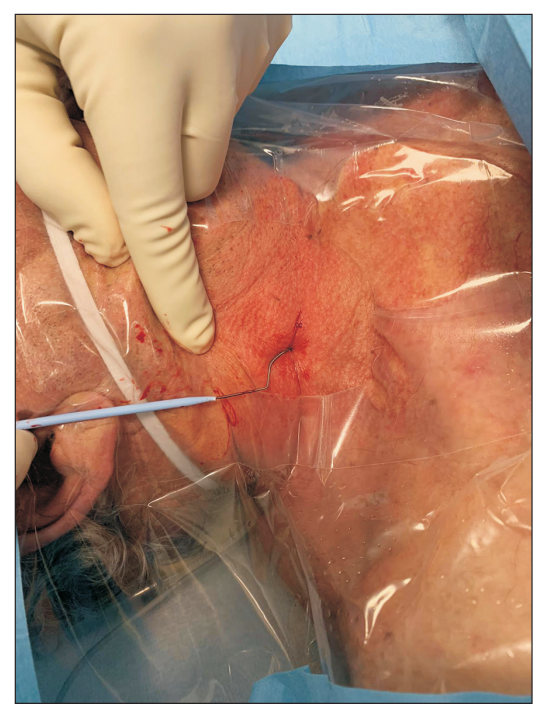

Fig. 4. Wire kinking and dilator tip fraying are seen after an attempt to advance though a poorly placed skin incision. Skin bridges require the operator to apply excessive force while trying to pass dilators or catheters, greatly increasing the risk of wire related complications.
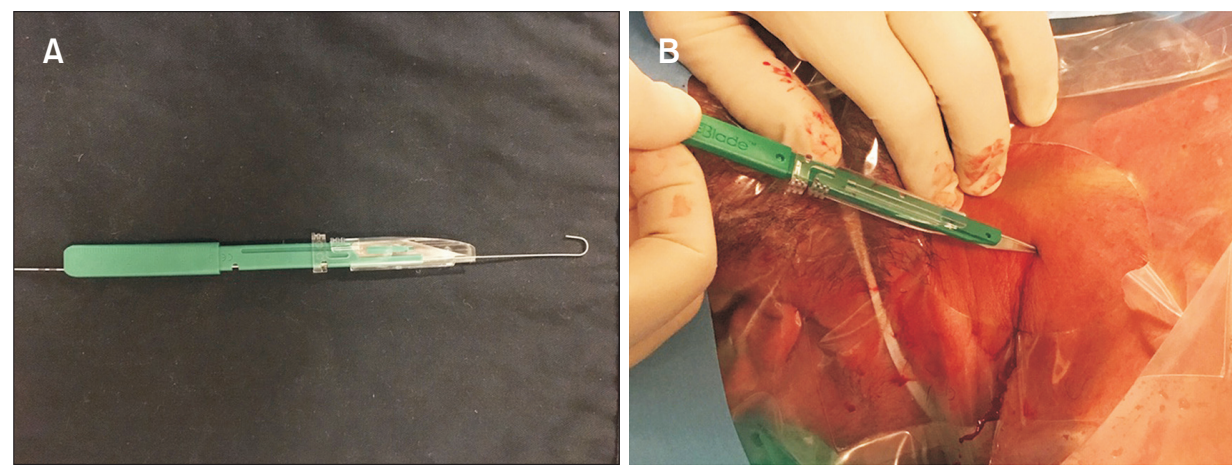

Fig. 3. (A) A wire threaded through the wire-guided scalpel channel and exiting the back of the scalpel handle. (B) A wireguided scalpel loaded on an insitu guidewire and advanced through the skin. By coupling the scalpel to the wire, the risk of misplaced incisions and residual skin bridges is eliminated. 
the entry site and the design of standard disposable scalpels that make proper parallel alignment with the wire difficult. In addition, bleeding at the wire entry site, skin folds, and the use of personal protective equipment like face shields may obscure visual fields during the incision. Finally, patient movement and suboptimal positioning, as is often encountered during emergency CVC placement, may further complicate precise skin incision placement.

Some of the more common complications encountered during CVC placement include local hematoma, inadvertent arterial puncture, and arrhythmia. Complications related to an indwelling CVC include central line-associated bloodstream infection and thrombus formation [5]. While less commonly reported, vascular guidewire and dilator-related complications such as wire fracture [6], wire loss [7], wire knotting [8], and major vascular injury often require immediate intervention and carry a significant risk of morbidity and mortality [9]. In our experience, poorly placed skin incisions often lead directly to wire kinking (Fig. 4), as excessive force used to push dilators or catheters through residual skin tags exceeds the tensile strength of the guidewire. The use of excessive force and wire kinking is the precipitating event leading to wire fracture and wire knotting during CVC placement and is likely an important factor in some cases of wire loss and major vascular injury [10].

The wire-guided scalpel enables clinicians to utilize the in situ guidewire to perform skin incisions with precision, even under suboptimal conditions. Elimination of skin bridges potentially decreases mechanical complications and damage to components of CVC insertion kits, such as guidewires, dilators, and catheters. While the direct cost of the wire-guided scalpel is greater than a standard safety scalpel, the above listed benefits likely make the wire-guided scalpel cost-effective.

An alternate technique for eliminating residual skin bridges is to make the incision prior to needle insertion and inserting the needle through the incision. This technique has several drawbacks, including bleeding from the incision site during needle and wire insertion and the risk of a misplaced incision, particularly during placement in awake subjects where patient movement may lead to significant changes in the target insertion site from the time of incision until the time of needle placement. In addition, the wire-guided scalpel carries the benefit of allowing the clinician to precisely incise the subcutaneous tissue surrounding the guidewire, further facilitating dilator and catheter insertion.

\section{CONCLUSION}

By utilizing the in-situ guidewire to direct the incision, the wire-guided scalpel allows for precise skin incision placement, even under suboptimal conditions. The routine use of a wire-guided scalpel essentially eliminates the risk of misaligned skin incisions and may significantly decrease the risk of potentially catastrophic wire-and dilator-related complications.

\section{CONFLICTS OF INTEREST}

JJR is patent holder of the device mentioned, Guideblade and has stock in company. The other authors have nothing to disclose.

\section{ORCID}

\author{
Jacob Jeffrey Rauchwerger \\ https://orcid.org/0000-0002-0768-0817 \\ Michael Serle \\ https://orcid.org/0000-0002-9943-9579 \\ Jeffrey C. Astbury \\ https://orcid.org/0000-0002-4956-016X
}

\section{AUTHOR CONTRIBUTIONS}

Concept and design: JJR. Analysis and interpretation: JJR. Data collection: none. Writing the article: all authors. Critical revision of the article: all authors. Final approval of the article: all authors. Statistical analysis: none. Obtained funding: none. Overall responsibility: all authors.

\section{REFERENCES}

1) Ruesch S, Walder B, Tramèr MR. Complications of central venous catheters: internal jugular versus subclavian access--a systematic review. Crit Care Med 2002;30:454-460.

2) Gershengorn HB, Garland A, Kramer
A, Scales DC, Rubenfeld G, Wunsch

$H$. Variation of arterial and central venous catheter use in United States intensive care units. Anesthesiology 2014;120:650-664.

3) Noel-Lamy M. The seldinger tech- nique: a short history, and its applications 60 years later. Univ Toronto Med J 2015;93:30-31.

4) Frogel JK, Rauchwerger JJ. Importance of dermatotomy incision without residual skin bridge during central 
venous catheterization. Vasc Specialist lnt 2020;36:122-123.

5) Patel AR, Patel AR, Singh S, Singh $S$, Khawaja 1. Central line catheters and associated complications: a review. Cureus 2019;11:e4717.

6) Park SK, Yi IK, Lee JH, Kim DH, Lee SY. Fracture of J-tipped guidewire during central venous catheterization and its successful removal under fluo- roscopic guidance- a case report. Korean J Anesthesiol 2012;63:457-460.

7) Srivastav R, Yadav V, Sharma D, Yadav V. Loss of guide wire: a lesson learnt review of literature. J Surg Tech Case Rep 2013;5:78-81.

8) Khan KZ, Graham D, Ermenyi A, Pillay WR. Case report: managing a knotted Seldinger wire in the subclavian vein during central venous cannulation.
Can J Anaesth 2007;54:375-379.

9) Bodenham A. Reducing major procedural complications from central venous catheterisation. Anaesthesia 2011;66:6-9.

10) Collier PE. Prevention and treatment of dilator injuries during central venous catheter placement. J Vasc Surg Venous Lymphat Disord 2019;7:789792. 